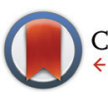

CrossMark \& click for updates

Cite this: Polym. Chem., 2016, 7. 6413

Received 19th August 2016, Accepted 29th September 2016 DOI: 10.1039/c6py01453d www.rsc.org/polymers

\title{
Narrow bandgap thienothiadiazole-based conjugated porous polymers: from facile direct arylation polymerization to tunable porosities and optoelectronic properties $\uparrow$
}

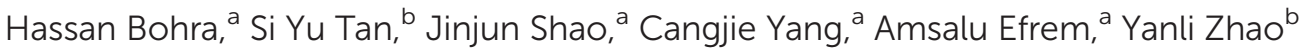 \\ and Mingfeng Wang*a
}

Conjugated porous polymers with narrow bandgaps are important for light harvesting in the near infrared region, but are limited by the availability of the appropriate building blocks and synthetic tools. Here we report a series of narrow bandgap conjugated porous polymers synthesized by facile direct arylation polymerization of thiophene-flanked thienothiadiazole with multibrominated monomers with different geometries. The polymer products show strong light absorption in the near infrared region, corresponding to narrow optical bandgaps below $1.3 \mathrm{eV}$. Under the same polymerization conditions, the morphologies, porosities and optoelectronic properties of the resulting polymers are determined by the chemical structures of the aryl bromides. The synthetic protocol of direct arylation polymerization and the structure-property relationship established in these narrow bandgap conjugated porous polymers will be important for rational material design towards applications such as gas separation/storage and photocatalysis.

\section{Introduction}

Conjugated porous polymers $(\mathrm{CPPs})^{1-5}$ integrated with high porosity, optoelectronic properties and chemical stability are a class of multifunctional organic materials that have attracted much attention owing to their potential applications such as gas storage, ${ }^{6-8}$ chemosensing, ${ }^{9,10}$ and photocatalysis. ${ }^{11-13}$ Similar to linear conjugated polymers, the design and synthesis of CPPs follows a modular approach. ${ }^{14}$ With careful selection of monomers and polymerization techniques, tunability over a wide range of physical and chemical properties can be achieved..$^{15,16}$ In particular, bandgap control is crucial in polymers for energy conversion as well as other lightharvesting applications. For instance, incorporation of strong electron-accepting moieties such as benzothiadiazoles and weak electron-donating units such as benzenes and fluorenes

\footnotetext{
${ }^{a}$ School of Chemical and Biomedical Engineering, Nanyang Technological University, 62 Nanyang Dr., Singapore 637459, Singapore.E-mail: mfwang@ntu.edu.sg ${ }^{b}$ Division of Chemistry and Biological Chemistry, School of Physical and Mathematical Sciences, Nanyang Technological University, 21 Nanyang Link, Singapore 637371, Singapore

$\dagger$ Electronic supplementary information (ESI) available: Mass spectra and cyclic voltammograms of TTD-(1-3). TGA, PXRD patterns, BET specific surface area plots and NL-DFT pore size distribution of CPP-(1-3). See DOI: 10.1039/ c6py01453d
}

in conjugated polymer networks not only reduced the bandgaps, but also improved the photocatalytic activity compared to pure electron-donating networks. ${ }^{17}$ A similar donor-acceptor (D-A) strategy was used recently by Bonillo et al. to finely tune the bandgap and emission properties of polyphenylene networks by modifying the monomer ratios of different electron-accepting moieties in a Suzuki-Miyaura polymerization protocol. ${ }^{18}$

Traditional synthesis of CPPs involving the aryl C-C bond formation has been carried out by transition-metal catalyzed reactions such as Sonogashira-Hagihara coupling, ${ }^{19}$ Yamamoto coupling, ${ }^{20}$ Suzuki-Miyaura coupling, ${ }^{16,21}$ and Stille coupling. ${ }^{22}$ Linker-specific polycondensation reactions such as Schiff-base condensation ${ }^{23}$ and imidization ${ }^{24}$ reactions have also been vastly utilized in the synthesis of CPPs. Most of these reactions, nevertheless, involve multiple synthetic steps for the functionalization of comonomers. Moreover, some transition-metal catalyzed reactions such as Stille coupling result in the formation of toxic byproducts which are detrimental to the environment.

Direct arylation polymerization (DAP) as an alternative to conventional polymerization reactions for the synthesis of linear $\pi$-conjugated polymers has attracted increasing attention in the last decade. ${ }^{25}$ DAP, which involves the facile onestep coupling of aryl bromides with $\mathrm{C}-\mathrm{H}$ active arenes without preactivation of $\mathrm{C}-\mathrm{H}$ bonds using flammable and/or toxic 
organometallic reagents, has enabled the synthesis of a myriad of linear conjugated polymers with qualities (regioselectivity, molecular weights, etc.) comparable to or even better than those synthesized by conventional coupling techniques. ${ }^{26-29}$ However, DAP as a synthetic tool for conjugated porous polymers has not been investigated until recently. ${ }^{30}$ For instance, Liu et al. ${ }^{30 a}$ have reported DAP between 1,2,4,5-tetrafluorobenzene and two aryl bromides, 1,3,5-tribomobenzene and tetrakis(4-bromophenyl)methane. Although the resulting polymers showed specific surface areas as high as $1170 \mathrm{~m}^{2} \mathrm{~g}^{-1}$, their wide bandgaps limited light absorption in visible and near infrared (NIR) regions. More recently, our group reported a facile one-step DAP using dibromothienophenanzine as a single monomer, resulting in CPPs with a narrow bandgap of $1.5 \mathrm{eV}$ and hierarchical porosity. ${ }^{30 b}$ However, the scope of CPPs that could be synthesized using the facile DAP scheme remains rather limited.

Herein, we report the synthesis of a series of narrowbandgap conjugated porous polymers by DAP of 4,6-di(2thienyl)thieno[3,4-c][1,2,5]thiadiazole (TTD) with multi-topic aryl bromides. TTD, composed of a strong electron-accepting core of thienothiadiazole flanked by thiophene groups on both sides, is a coplanar $\pi$-conjugated narrow bandgap monomer. We speculated that the electron-deficient nature of the thienothiadiazole core could result in effective cleavage of the acidic $\mathrm{C}-\mathrm{H}$ bonds in the thiophene groups. To understand the reactivity and regioselectivity of $\mathrm{TTD}, \mathrm{C}-\mathrm{H}$ direct arylation coupling between TTD and three monobrominated reagents (MBr-1-3, Scheme 1) was examined, resulting in three donoracceptor-donor (D-A-D) triads that are easier to purify and characterize than CPPs. Then three representative electronrich/neutral aryl bromides, 1,3,5-tribromobenzene, ${ }^{15 a, 17}$ 2,2',7,7'-tetrabromo-9,9'-spirobifluorene ${ }^{20 a, 31}$ and tris(4-bromophenyl)amine ${ }^{32}$ were used as comonomers in DAP with TTD. The structure-property relationships of TTD-based CPPs were studied through systematic characterization using nuclear magnetic resonance (NMR) spectroscopy, Fourier transform infrared spectroscopy (FTIR), elemental analysis, electron microscopy, porosity analysis and UV-VIS-NIR absorption spectroscopy.

\section{Experimental}

\section{General synthesis procedure for TTD-Xs via direct arylation coupling}

TTD (80 mg, $0.2614 \mathrm{mmol}$ ), MBr-1, -2 or -3 (2.61 mmol), $\mathrm{Pd}_{2}(\mathrm{dba})_{3} \quad(0.013 \mathrm{mmol}, 11.9 \mathrm{mg}),(o-\mathrm{MeOPh})_{3} \mathrm{P} \quad$ (0.02614, $9.2 \mathrm{mg}), \quad \mathrm{K}_{2} \mathrm{CO}_{3}(1.045 \mathrm{mmol}, 144.3 \mathrm{mg})$ and $\mathrm{PivOH}$ (0.137 mmol, $13.3 \mathrm{mg}$ ) were added into a reaction vial equipped with a magnetic stirring bar. $o$-Xylene $(1.3 \mathrm{ml}, 0.2 \mathrm{M})$ was added inside a glovebox and the vial was sealed. The reaction was carried out for 24 hours in an oil bath preheated to $120{ }^{\circ} \mathrm{C}$. After completion, the reaction mixture was diluted with $30 \mathrm{~mL}$ chloroform and filtered to remove any insoluble matter. Solvent was removed under vacuum and the crude product was separated over silica gel. The pure product was obtained by precipitation in methanol.

TTD-1: MBr-1 = 1-bromo-4-hexylbenzene (629.4 mg). Yield: $110.9 \mathrm{mg}(95 \%) .{ }^{1} \mathrm{H} \mathrm{NMR}\left(\mathrm{CDCl}_{3}, 300 \mathrm{MHz}\right) \delta \mathrm{ppm}, 7.57$ $(\mathrm{d}, 4 \mathrm{H}, J=8.3 \mathrm{~Hz}), 7.54(\mathrm{~d}, 2 \mathrm{H}, J=4.1 \mathrm{~Hz}), 7.30(\mathrm{~d}, 2 \mathrm{H}, J=$ $4.0 \mathrm{~Hz}), 7.21(\mathrm{~d}, 4 \mathrm{H}, J=8.3 \mathrm{~Hz}), 2.69-2.58(\mathrm{~m}, 4 \mathrm{H}), 1.62(\mathrm{~d}, 4 \mathrm{H}$, $J=7.9 \mathrm{~Hz}), 1.62(\mathrm{~s}, 12 \mathrm{H}), 0.89(\mathrm{t}, 6 \mathrm{H}, J=6.9 \mathrm{~Hz})$. EI MS $m / z(\%)$ $626\left(\mathrm{M}^{+}, 93\right), 605$ (10), 568 (8), 499 (13).

TTD-2: MBr-2 = 4-bromotriphenylamine (846.1 mg). Yield: $130.6 \mathrm{mg}(80 \%) .{ }^{1} \mathrm{H} \mathrm{NMR}\left(\mathrm{CDCl}_{3}, 300 \mathrm{MHz}\right) \delta \mathrm{ppm}, 7.55-7.52$ $(\mathrm{m}, 4 \mathrm{H}), 7.50(\mathrm{~d}, 2 \mathrm{H}, J=2.0 \mathrm{~Hz}), 7.54(\mathrm{~d}, 2 \mathrm{H}, J=4.1 \mathrm{~Hz})$, $7.31(\mathrm{~d}, 2 \mathrm{H}, J=2.0 \mathrm{~Hz}), 7.29(\mathrm{~d}, 4 \mathrm{H}, J=1.2 \mathrm{~Hz}), 7.24(\mathrm{~d}, 2 \mathrm{H}, J=$ $7.9 \mathrm{~Hz}), 7.16-7.02(\mathrm{~m}, 18 \mathrm{H})$. EI MS $m / z(\%) 792\left(\mathrm{M}^{+}, 100\right)$, 644 (47), 605 (8), 456 (10).

TTD-3: $\mathrm{MBr}-3$ = 2-bromo-9,9-dioctyl-9H-fluorene (1.22 g). Yield: $121 \mathrm{mg}$ (83\%). ${ }^{1} \mathrm{H} \mathrm{NMR}\left(\mathrm{CDCl}_{3}, 300 \mathrm{MHz}\right) \delta \mathrm{ppm}, 7.57$ (s, 6H, br), 7.61 (s, 4H, br), 7.42 (d, 2H, $J=3.8 \mathrm{~Hz}$ ), 7.35 (s, 6H, br), 2.07-1.92 (m, 8H), 1.22-0.98 (m, 40H), $0.8(\mathrm{t}, 12 \mathrm{H}$,
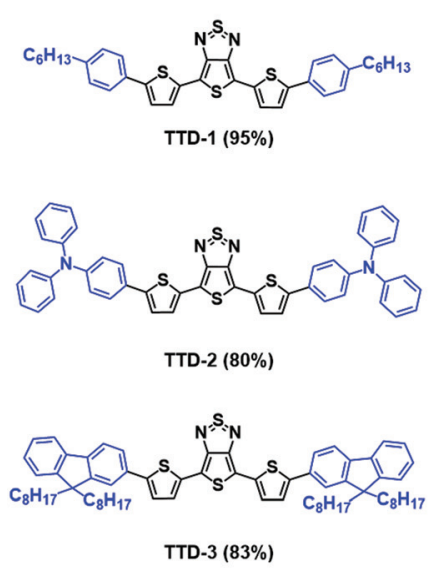
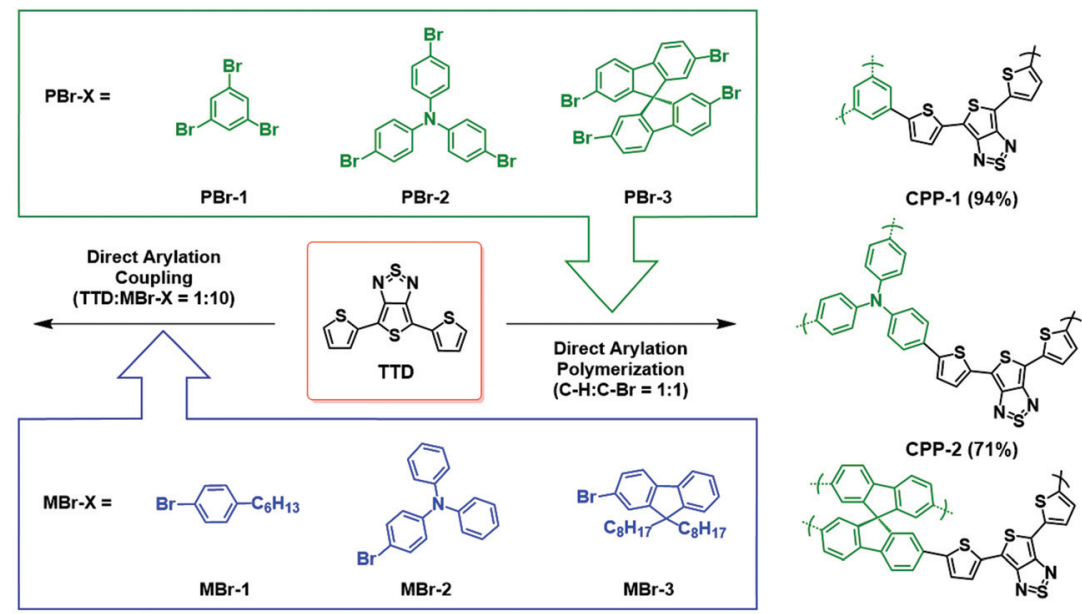

CPP-3 (79\%)

Scheme 1 Synthesis of TTD-based D-A-D triads and CPPs by direct arylation coupling. 
$J=1 \mathrm{~Hz}), 0.64(\mathrm{~s}, 8 \mathrm{H}, \mathrm{br})$. EI MS m/z (\%) $1083\left(\mathrm{M}^{+}, 100\right), 1060$ (8), 897.97 (6), 855 (21).

\section{General synthesis procedure for CPP-Xs via direct arylation polymerization}

TTD, PBr-X (C-H:C-Br = 1:1), $\operatorname{Pd}_{2}(\mathrm{dba})_{3} \quad(5 \mathrm{~mol} \%)$, $(o-\mathrm{MeOPh})_{3} \mathrm{P} \quad(10 \mathrm{~mol} \%), \mathrm{K}_{2} \mathrm{CO}_{3}(400 \mathrm{~mol} \%)$ and $\mathrm{PivOH}$ (50 mol\%) were added to a reaction vial charged with a magnetic stirring bar. $o$-Xylene $(0.2 \mathrm{M})$ was added inside a glovebox and the vial was sealed with a rubber cap. The reaction was carried out at $120{ }^{\circ} \mathrm{C}$ in an oil bath for 48 hours. After cooling to room temperature, the reaction mixture was diluted with THF and filtered under vacuum. The solids were washed with methanol, water, dilute $\mathrm{HCl}$ and $\mathrm{THF}$ and then subjected to Soxhlet extraction with methanol and THF for 24 hours, respectively. The residual solids were collected and dried at $100{ }^{\circ} \mathrm{C}$ under vacuum to obtain the final product.

CPP-1: 1,3,5-tribromobenzene (70 mg), TTD (102 mg), $\mathrm{Pd}_{2}(\mathrm{dba})_{3} \quad(15.27 \mathrm{mg}), \quad(o-\mathrm{MeOPh})_{3} \mathrm{P} \quad(11.74 \quad \mathrm{mg}), \quad \mathrm{K}_{2} \mathrm{CO}_{3}$ (184.37 mg), PivOH (17.14 mg) and $o$-xylene $(1.67 \mathrm{~mL})$. Yield: $110.9 \mathrm{mg}$ (94\%). IR (KBr cm${ }^{-1}$ ): 3059 (br), 1583 (sh), 1555 (sh), 1491 (sh), 1408 (sh), 856 (sh), 833 (sh), 792 (br). Elemental Analysis (\%) for $\mathrm{C}_{48} \mathrm{H}_{18} \mathrm{~N}_{6} \mathrm{~S}_{12}$ Calculated: C, 54.24; $\mathrm{H}, 1.69 ; \mathrm{N}$, 7.91; S, 36.16. Found: C, 51.34; H, 1.88; N, 5.89; S, 26.05.

CPP-2: tris(4-bromophenyl)amine (126 mg), TTD (120 mg), $\mathrm{Pd}_{2}(\mathrm{dba})_{3} \quad(17.95 \mathrm{mg}), \quad(o-\mathrm{MeOPh})_{3} \mathrm{P} \quad(13.816 \mathrm{mg}), \mathrm{K}_{2} \mathrm{CO}_{3}$ (216.75 mg), PivOH (20.15 mg) and $o$-xylene $(1.96 \mathrm{~mL})$. Yield: $130.6 \mathrm{mg}$ (71\%). IR (KBr cm ${ }^{-1}$ ): 3054 (br), 3021 (sh), 1593 (sh), 1529 (sh), 1480 (sh), 1429 (br), 1320 (sh), 1266 (br), 847 (br), 829 (sh), 792 (br). Elemental Analysis (\%) for $\mathrm{C}_{72} \mathrm{H}_{36} \mathrm{~N}_{8} \mathrm{~S}_{12}$ Calculated: C, 61.89; H, 2.58; N, 8.02; S, 27.51. Found: C, $58.65 ; \mathrm{H}, 2.576 ; \mathrm{N}, 6.468 ; \mathrm{S}, 21.09$.

CPP-3: 2,2',7,7'-tetrabromo-9,9'-spirobifluorene (105.4 mg), TTD (102.06 mg), $\quad \operatorname{Pd}_{2}(\mathrm{dba})_{3} \quad(15.27 \mathrm{mg}), \quad(o-\mathrm{MeOPh})_{3} \mathrm{P}$ (11.74 mg), $\mathrm{K}_{2} \mathrm{CO}_{3}(184.37 \mathrm{mg}), \mathrm{PivOH}(17.14 \mathrm{mg})$ and $o$-xylene (1.67 mL). Yield: $121 \mathrm{mg}$ (79\%). IR (KBr cm $\left.{ }^{-1}\right): 3058$ (sh), 1636 (br), 1600 (sh), 1572 (sh), 1527 (br), 1435 (sh), 1410 (sh), 1249 (sh), 1139 (br), 1055 (sh), 854 (sh), 792 (br). Elemental Analysis (\%) for $\mathrm{C}_{49} \mathrm{H}_{20} \mathrm{~N}_{4} \mathrm{~S}_{8}$ Calculated: C, 63.88; H, 2.19; N, 6.08; S, 27.85. Found: C, 57.26; H, 1.98; N, 4.4; S, 19.93.

\section{Results and discussion}

The synthetic routes to the TTD-based small molecular D-A-D triads and CPPs are shown in Scheme 1. These triads serve as model compounds for the respective conjugated polymer networks. A $\operatorname{Pd}(0)$ catalyst, $\operatorname{Pd}_{2}(\mathrm{dba})_{3}$, in combination with $(o-\mathrm{MeOPh})_{3} \mathrm{P}$ was used in a carboxylate-mediated direct arylation coupling to produce TTD-(1-3). ${ }^{28}$ The reaction was carried out at $120{ }^{\circ} \mathrm{C}$ for 24 hours. All triads were obtained in high yields - 95\% for TTD-1, 80\% for TTD-2 and 83\% for TTD-3. ${ }^{1} \mathrm{H}$-NMR spectra of the triads (Fig. 1) show the peak from the $\beta$-proton of the TTD core at $7.30 \mathrm{ppm}$ in TTD-1, $7.31 \mathrm{ppm}$ in TTD-2 and 7.41 ppm in TTD-3, respectively, suggesting good regioselectivity of TTD under the present reaction conditions.
(A)
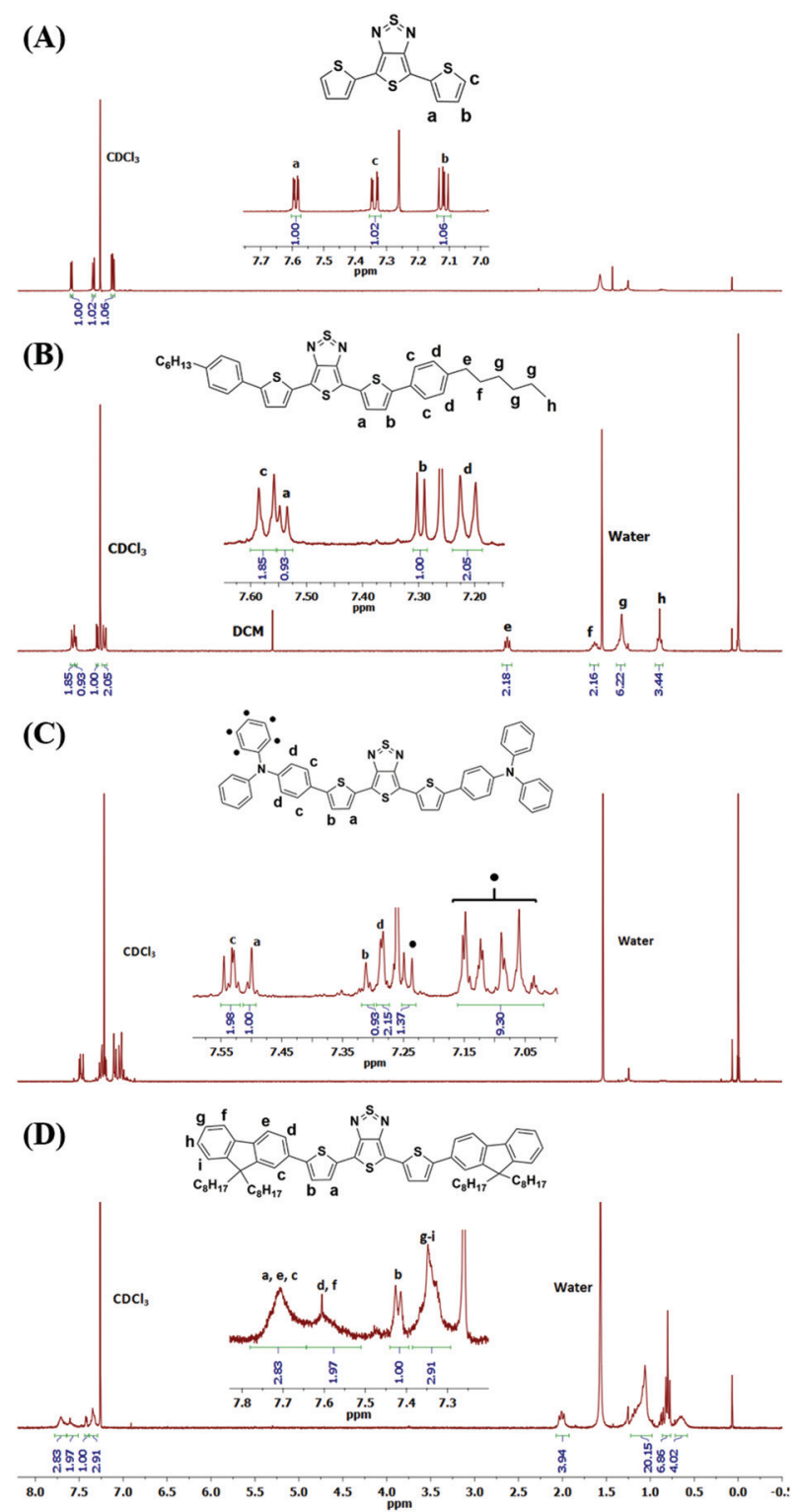

Fig. $1{ }^{1} \mathrm{H}-\mathrm{NMR}$ spectra of (A) TTD, (B) TTD-1, (C) TTD-2 and (D) TTD-3 in $\mathrm{CDCl}_{3}$ at $298 \mathrm{~K}$ with complete assignment of protons.

The chemical structures of the triads were further confirmed by mass spectroscopy (Fig. $\mathrm{S} 1 \dagger$ ). The relatively high reactivity and regioselectivity of TTD observed in these model reactions are crucial for the following DAP to obtain structurally defined CPPs.

Optical properties of TTD-based triads were studied in chlorobenzene by UV-Vis-NIR absorption spectroscopy (Fig. 2). Absorption maxima of all triads show a significant red shift when compared to the spectrum of TTD, which is mainly attributed to the donor-acceptor interaction between TTD and its donor partner. It can be observed that the extent of the redshift is proportional to the electron-donating strength of the donor. Among the three D-A-D triads, TTD-2-TTD coupled with two triphenylamine moieties, absorbs at the longest wavelength and has the smallest optical bandgap $\left(E_{\mathrm{g}}^{\mathrm{opt}}=1.38 \mathrm{eV}\right)$, 


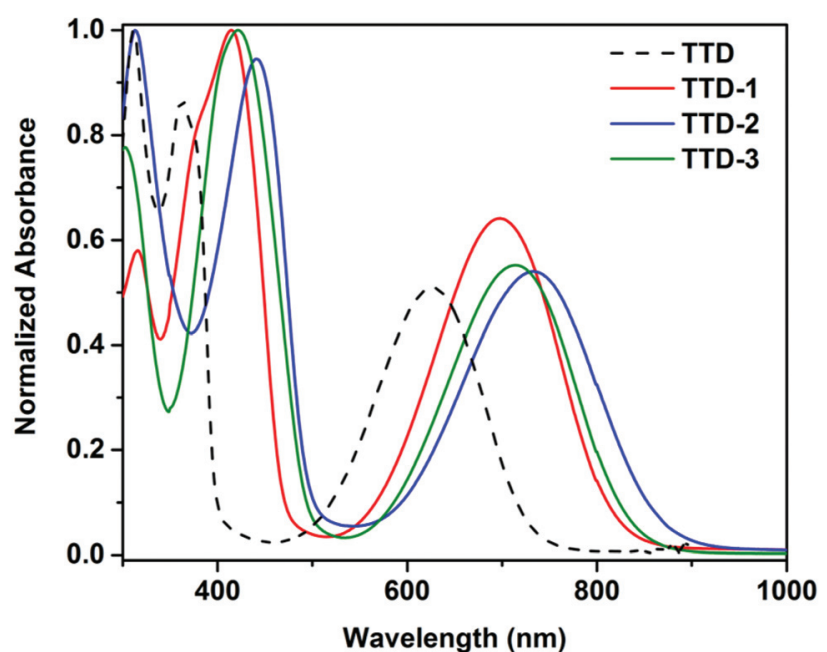

Fig. 2 UV-Vis-NIR absorption spectra of TTD-(1-3) in chlorobenzene solutions.

whereas TTD-1 consisting of phenyl groups has the largest optical bandgap $\left(E_{\mathrm{g}}^{\mathrm{opt}}=1.48 \mathrm{eV}\right)$. The decrease of the bandgap in the order of TTD- $2<$ TTD- $3<$ TTD- 1 is consistent with the increasing strength of the electron donating groups (triphenylamine > fluorene > phenyl) in the triads.

TTD-based near infrared conjugated porous polymers (CPP(1-3)) were synthesized using the same reaction conditions as for the synthesis of TTD-1-3 triads, with the exception of the reaction time and monomer ratio. The $\mathrm{DAP}^{28}$ reaction condition used here was found efficient for the synthesis of benzodithiophene-based linear polymers and the $\mathrm{C}-\mathrm{H}$ activation of a variety of thiophene-flanked acceptor molecules ${ }^{29 a-d}$ including thienothiadiazole ${ }^{29 e}$ for the synthesis of donor-acceptor linear polymers. A molar ratio of $1: 1$ between $\mathrm{C}-\mathrm{H}$ and $\mathrm{C}-\mathrm{Br}$ bonds of the monomers was maintained and all the polymerization reactions were carried out for 48 hours to ensure as complete a reaction as possible. Polymers were purified by washing thoroughly with organic (methanol, chloroform and THF) and aqueous solvents (water and diluted $\mathrm{HCl}$ ) to remove all oligomeric products as well as any byproducts of DAP. The CPPs obtained here appear as green powders that are insoluble in common organic solvents such as THF, chloroform, toluene and chlorobenzene.

Characterization of the chemical structures of CPP-(1-3) was first carried out using FTIR spectroscopy (Fig. 3). A band at $1489 \mathrm{~cm}^{-1}$ in the FTIR spectra of TTD, which is assigned to the symmetric stretch of the aromatic $\mathrm{C}=\mathrm{C}$ bond of thiophene, is clearly present in the spectra of all CPPs. The symmetric $\mathrm{C}=\mathrm{C}$ stretching bands of their respective aryl bromide comonomers appear at $1583 \mathrm{~cm}^{-1}$ in CPP-1, $1480 \mathrm{~cm}^{-1}$ in CPP-2 and $1465 \mathrm{~cm}^{-1}$ in CPP-3, respectively. Stretching vibrations of thiophene rings in TTD were also detected as weak bands in the frequency range between 840 and $860 \mathrm{~cm}^{-1}$. CPP-2 showed the characteristic stretching bands of aromatic $\mathrm{C}-\mathrm{N}$ bonds at $1320 \mathrm{~cm}^{-1}$ (Fig. 3b). The obvious attenuation of the $\mathrm{C}-\mathrm{Br}$ stretch band at $1096 \mathrm{~cm}^{-1}$ in CPP-1 suggests the a)

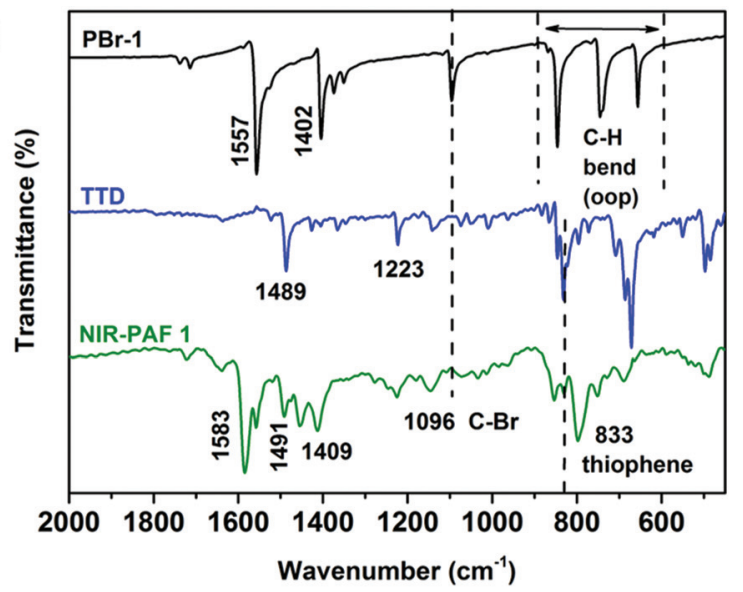

b)

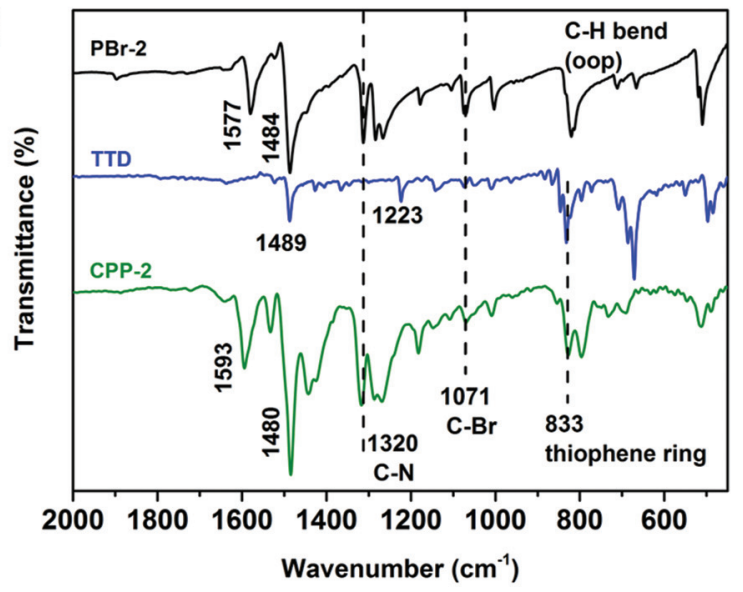

c)

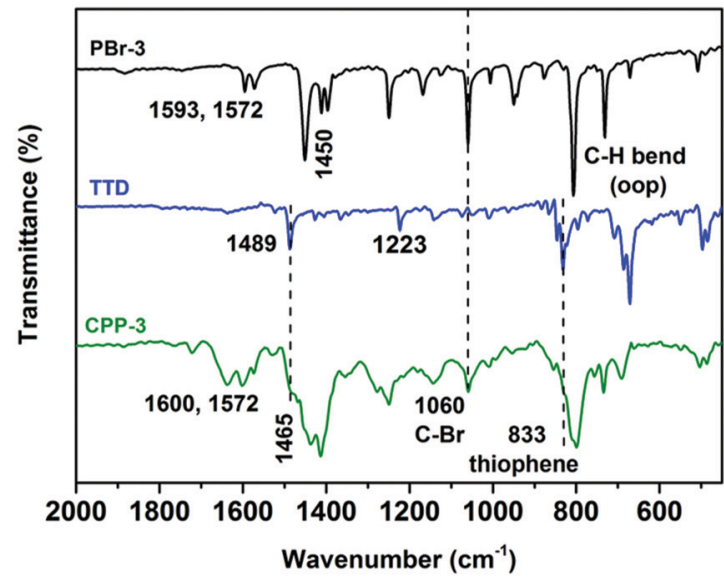

Fig. 3 Comparison of FT-IR spectra (fingerprint region) of (a) CPP-1, (b) CPP-2 and (c) CPP-3 shown in green with TTD (blue) and their respective aryl bromide comonomers (black).

highly efficient direct arylation reaction and the large extent of polymerization. In contrast to CPP-1, small peaks at 1071 and $1060 \mathrm{~cm}^{-1}$ corresponding to the $\mathrm{C}-\mathrm{Br}$ stretch band were still observed in CPP-2 and CPP-3, respectively, suggesting the lower extent of polymerization in the latter two polymers than the former. 
CPP-(1-3) were further characterized by solid state ${ }^{13} \mathrm{C} \mathrm{CP} /$ MAS NMR spectroscopy to confirm their structures. ${ }^{13} \mathrm{C}$ NMR spectra of all polymers with complete peak assignments are shown in Fig. 4. A sharp peak around $156 \mathrm{ppm}$ and a broad peak around $112 \mathrm{ppm}$, which are assigned to the carbons of the thienothiadiazole moiety of TTD, are observed in the spectra of all CPPs. In CPP-1, the broad peaks observed at 141.7 and 135.5 ppm could be attributed to the substituted carbons of the TTD ( $\alpha$-carbon) and the phenyl repeating unit, respectively (Fig. 4a). In addition, the peaks of unsubstituted carbons $(\mathrm{C}-\mathrm{H})$ of these monomers overlap into a broad peak located at $125.8 \mathrm{ppm}$. In CPP-2, the carbon signal from the tertiary amine $(\mathrm{C}-\mathrm{N})$ group appears at $145.8 \mathrm{ppm}$ and overlaps with the signals of the substituted carbons of triphenylamine and TTD units (Fig. 4b). Unsubstituted carbons of CPP-2 are assigned to the broad peak at $128 \mathrm{ppm}$ and its shoulder at 132.6 ppm. In CPP-3, the signals from the phenyl rings of spirobifluorene appear as broad peaks between 150 and 124 ppm, as shown in Fig. 4c. The sharp peak at $66.5 \mathrm{ppm}$ is assigned to the quaternary carbon of spirobifluorene. The ${ }^{13} \mathrm{C}-\mathrm{NMR}$ results discussed above, which are complementary to the FTIR results, further prove the chemical structures of the target CPP-1-3 obtained under the present direct arylation scheme.

Elemental analysis of CPPs showed some inconsistency between the theoretical (neglecting the contribution of bromide end groups) and experimental values, which is typical of highly conjugated organic networks. ${ }^{30 b}$ A better match between the theoretical and experimental mass percentages was observed in CPP-1 compared to CPP-2 and CPP-3. These results are consistent with the FTIR spectra of CPP-2 and CPP-3 (Fig. 3b and c) where the appearance of a weak C-Br band indicates their lower extent of polymerization.

Powder X-ray diffraction (XRD) patterns of all CPPs exhibit broad peaks in the range $2 \theta=5-60^{\circ}$, indicating the amorphous nature of these polymers (Fig. S3†). Thermogravimetric analysis shows that all CPPs (Fig. S4 $\dagger$ ) are stable up to $300{ }^{\circ} \mathrm{C}$ a)

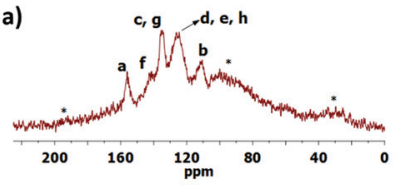

b)

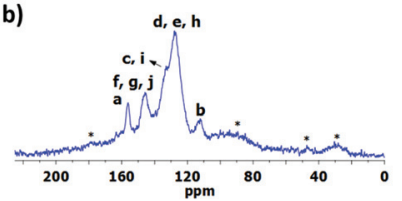

c)

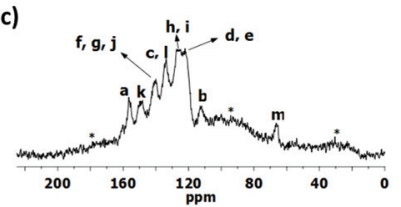

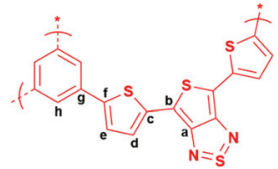
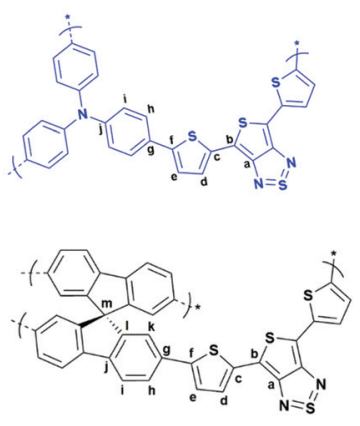

Fig. $4{ }^{13} \mathrm{C}$ CP/MAS spectra of CPP-1 (a), CPP-2 (b) and CPP-3 (c). under a nitrogen atmosphere, a characteristic of the good thermal stability of such covalent polymer networks.

Morphologies of CPP-(1-3) were studied by scanning electron microscopy (SEM) and transmission electron microscopy (TEM). From the SEM image of CPP-1 (Fig. 5), dense networks of thin fibres can be observed most likely due to aggregation of the individual strands. The thickness of the fibres estimated from the SEM images is $\sim 30 \mathrm{~nm}$ (Fig. S8a $\dagger$ ).

Both CPP-2 and CPP-3 appear as microspheres with different sizes as seen from their SEM images (Fig. 5b and c). The particles of CPP-3 are approximately twice as large as those of CPP-2. Moreover, the micro-spheres of CPP-2 have a smooth surface, in contrast to the granular and relatively rough surface of CPP-3 (Fig. S5c $\dagger$ ). While the high-resolution TEM images (Fig. 5d-f) clearly show the microporous structures of CPP-2 (Fig. 5e) and CPP-3 (Fig. 5f), the microporous nature is not obvious in CPP-1 (Fig. $5 \mathrm{~d}$ ), presumably due to its smaller pore sizes and tighter $\pi-\pi$ stacking caused by the relatively small and flat phenyl repeating unit in CPP-1.

Porosities of CPP-1, CPP-2 and CPP-3 were studied in physisorption experiments using nitrogen as the sorbate molecule. Sorption isotherms of CPPs measured at $77 \mathrm{~K}$ are shown in Fig. 6. All polymers show a combination of Type II and Type IV sorption isotherms according to IUPAC classification. The uptake of $\mathrm{N}_{2}$ at relatively low pressures $p / p_{\mathrm{o}}<0.1$ is much lower compared to previously reported porous organic polymers, ${ }^{1,6,14}$ suggesting the relatively low microporosity in these
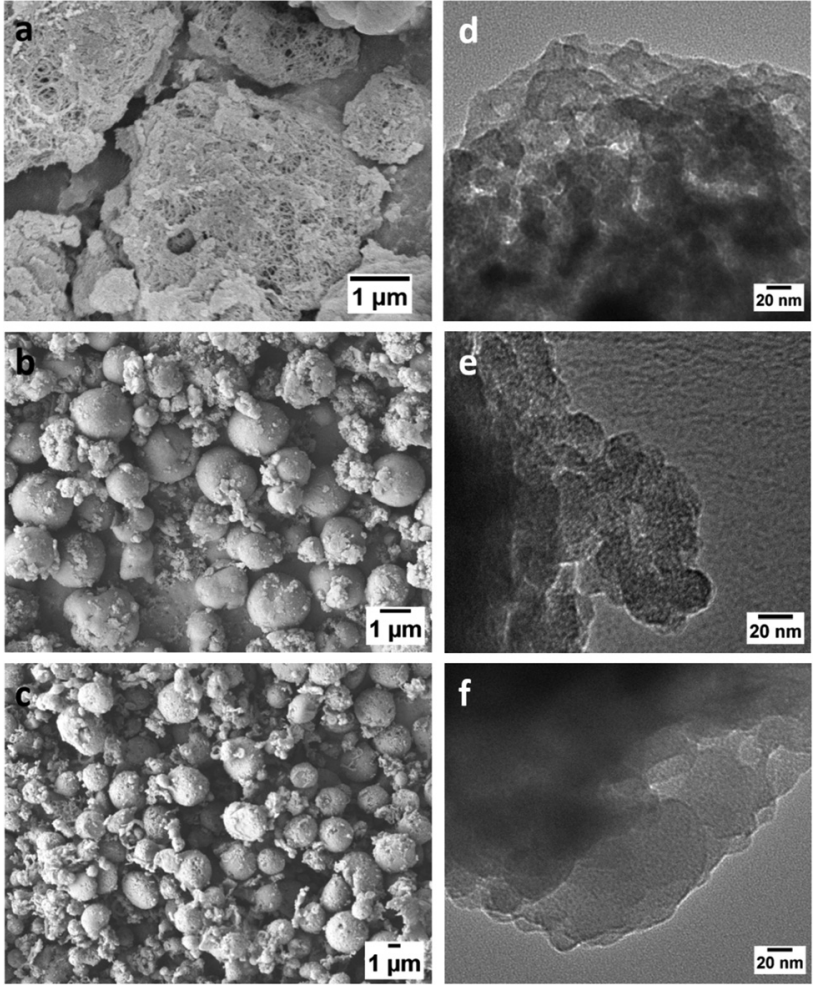

Fig. $5 \operatorname{SEM}(a, b, c)$ and $\operatorname{TEM}(d, e, f)$ images of CPP-1 $(a, d), C P P-2(b, e)$ and CPP-3 (c, f). 


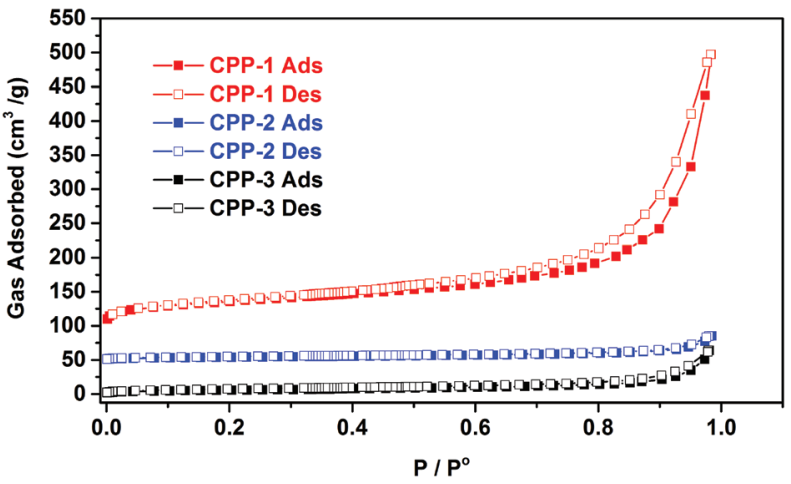

Fig. $6 \mathrm{~N}_{2}$ adsorption-desorption isotherms of CPP-(1-3) measured at $77 \mathrm{~K}$. For clarity, the isotherms of CPP-1 and CPP-2 were shifted vertically by $50 \mathrm{~cm}^{3} \mathrm{~g}^{-1}$, respectively.

polymers. In contrast, a sharp increase of $\mathrm{N}_{2}$-uptake was observed at $p / p_{\mathrm{o}}=0.9-1$, an indication of the presence of dominating meso- and macropores from the inter-particular voids in these polymers. A small degree of hysteresis was observed in the isotherms of CPP-1 in the relatively high pressure range $\left(0.5<p / p_{\mathrm{o}}<0.99\right)$. This can be explained by the presence of pores with narrow openings that restrict the access of nitrogen molecules. Alternatively, this could also be attributed to the swelling of the polymer framework due to the dissolution of the adsorbate into the non-porous sections of polymer during the adsorption cycle. ${ }^{33}$

Key porosity properties of CPPs are listed in Table 1. Among the three polymers, CPP-1 with fibrillar networks shows the largest Brunauer-Emmett-Teller (BET) surface area of $128.3 \mathrm{~m}^{2} \mathrm{~g}^{-1}$, which is significantly higher than those of the CPP-2 (14.2 $\left.\mathrm{m}^{2} \mathrm{~g}^{-1}\right)$ and CPP-3 (20.63 $\left.\mathrm{m}^{2} \mathrm{~g}^{-1}\right)$ microspheres. Pore size distribution (PSD) profiles of CPPs were calculated using the NL-DFT method (Fig. S7 $\dagger$ ). All CPPs have a broad PSD ranging from $14 \AA$ to well beyond $200 \AA$. The structureporosity relationship that we observed here in CPP-1-3 is similar to that in previously reported conjugated microporous polymers (CMPs). ${ }^{15,19,34}$ For instance, the lower surface area of CPP-2 as compared to CPP-1, which is consistent with the morphologies of these polymers as shown in the SEM images (Fig. 5), may be attributed to the larger size of the triphenylamine moiety. Cooper and co-workers also reported that

Table 1 N2 adsorption characteristics of CPP-(1-3)

\begin{tabular}{lllll}
\hline Species & $\begin{array}{l}S_{\mathrm{BET}}{ }^{a} \\
\left(\mathrm{~m}^{2} \mathrm{~g}^{-1}\right)\end{array}$ & $\begin{array}{l}S_{\mathrm{DFT}}{ }^{b} \\
\left(\mathrm{~m}^{2} \mathrm{~g}^{-1}\right)\end{array}$ & $\begin{array}{l}V_{\text {micro }}{ }^{c} \\
\left(\mathrm{~cm}^{3} \mathrm{~g}^{-1}\right)\end{array}$ & $\begin{array}{l}V_{\text {tot, DFT }} \\
\left(\mathrm{cm}^{3} \mathrm{~g}^{-1}\right)\end{array}$ \\
\hline CPP-1 & 128.3 & 95.36 & 0 & 0.52 \\
CPP-2 & 14.2 & 10.9 & 0 & 0.04 \\
CPP-3 & 20.63 & 17.2 & 0 & 0.08
\end{tabular}

${ }^{a}$ Specific surface area calculated from the adsorption branch of the $\mathrm{N}_{2}$ isotherm using the BET method. ${ }^{b}$ Specific surface area calculated using the NL-DFT method for slit pores with the equilibrium model. ${ }^{c}$ Micro-pore volume calculated from the adsorption branch of the $\mathrm{N}_{2}$ isotherm using the $t$-plot method. increasing the size of monomers in an A2 + B3 synthetic model led to polymers with lower surface areas. ${ }^{15 a}$

Optical properties of CPPs in chlorobenzene dispersions were studied by UV-Vis-NIR spectroscopy (Fig. 7). Compared to the absorption spectra of the D-A-D triads, all the three polymers (CPP-(1-3)) show a broad absorption across the visible and the near infrared regions. Despite some light scattering effect of the polymer dispersions which made it difficult to estimate the wavelength of the onset absorption, the bandedge absorption peaks of CPP-1-3 at 772, 840 and $727 \mathrm{~nm}$, respectively, remain well-resolved. The optical bandgaps of CPP-1-3 follow the trend of CPP-2 < CPP-1 $\approx \mathrm{CPP}-3$ (Table S1 $\dagger$ ). CPP-2 absorbs at the longest wavelength in the NIR region and has the smallest bandgap, largely attributed to the strong donor-acceptor interactions between triphenylamine and TTD. The slightly larger bandgap of CPP-3 vs. CPP-1 could be attributed to its lowest extent of polymerization as reflected from the FTIR spectrum (Fig. 3 and S2 $\dagger$ ).

Electrochemical properties of CPPs vs. TTD-based triads were evaluated by cyclic voltammetry (CV). While all triads showed reversible oxidation and quasi-reversible reduction peaks (Fig. S8†), irreversible oxidation and reduction peaks were observed in all the three CPPs (Fig. 8). Oxidation and reduction potentials of CPPs provide further insight into their electronic structures. Oxidation potential $\left(E_{\mathrm{ox}}\right)$ of CPP-3 at 0.74 $\mathrm{V}$ was the highest among the three CPPs, followed by CPP-1 $\left(E_{\mathrm{ox}}^{\text {onset }}=0.68 \mathrm{eV}\right)$ and CPP-2 $\left(E_{\mathrm{ox}}^{\text {onset }}=0.65 \mathrm{eV}\right)$. The HOMO levels of CPP-1-3 estimated from the CV results are -5.48 , -5.45 and $-5.54 \mathrm{eV}$, respectively. A similar trend was observed for the reduction potential as well, resulting in the LUMO levels which are $-4.07,-4.11$ and $-4.03 \mathrm{eV}$ for CPP-1-3, respectively. These results imply that the extended conjugation of TTD with an electron-donor led to a cathodic shift of the oxidation potentials and therefore an increase of their HOMO energy levels. The extent of the shift is proportional to the strength of the donor. As a consequence, the push-pull effect

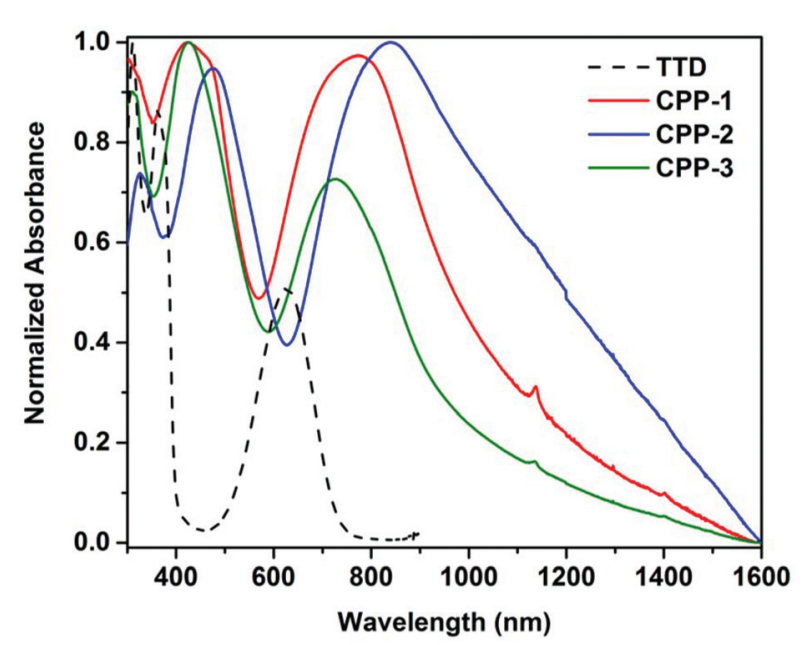

Fig. 7 UV-Vis-NIR absorption spectra of CPP-(1-3) in chlorobenzene dispersions. UV-Vis spectrum of TTD is plotted for comparison. 


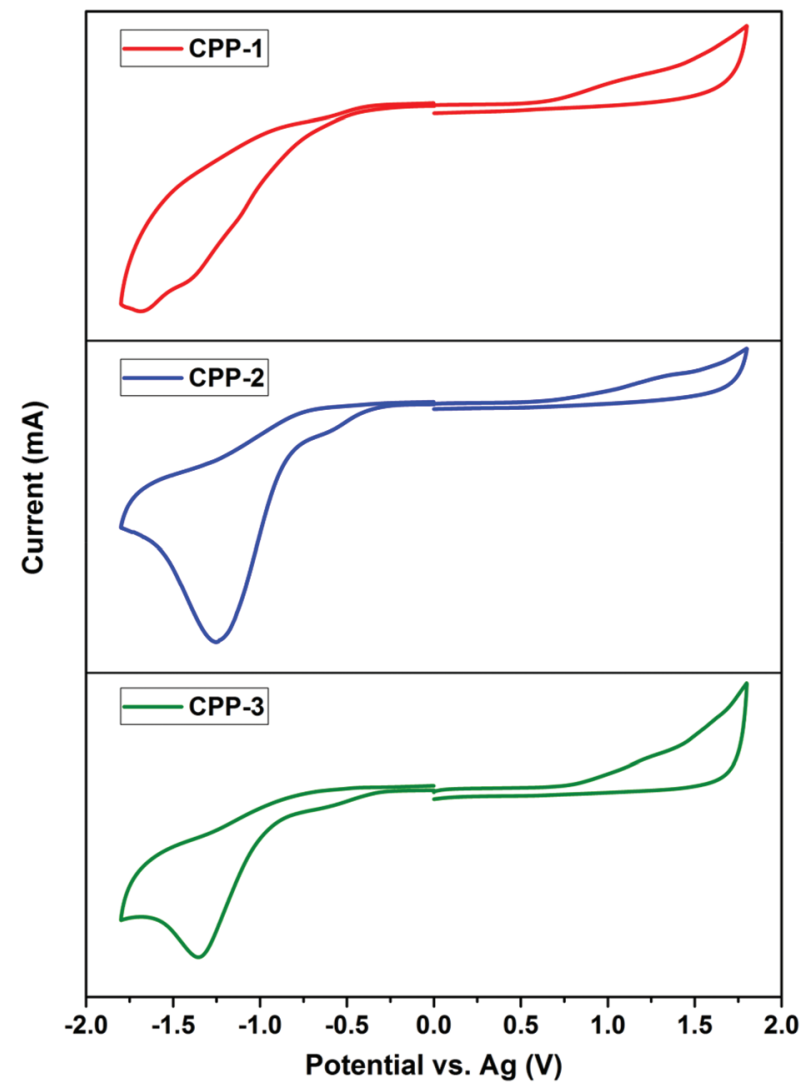

Fig. 8 Cyclic voltammograms of CPP-(1-3) in $0.1 \mathrm{M}$ solution of $\mathrm{Bu}_{4} \mathrm{NPF}_{6}$ in dry $\mathrm{CH}_{3} \mathrm{CN}$ as the supporting electrolyte.

between TTD and donor moieties results in a systematic narrowing of the HOMO-LUMO gaps of CPPs such that the electrochemical bandgaps follow the order of CPP- $<$ CPP-1 < CPP-3 (Table 2). The larger bandgap and the lower HOMO energy level of CPP-3 compared to CPP-1 can be explained by the lower degree of polymerization in the former. These results are consistent with the optical bandgaps of these CPP polymers.

While TTD-based linear narrow-bandgap polymers have shown potential applications for organic NIR photodetectors and field effect transistors, ${ }^{35}$ we expect that the 2D/3D TTDconjugated porous networks described above would find applications such as photocatalysis to harvest light in the NIR region. Moreover, incorporation of strong acceptor molecules in polymer networks of $\pi$-conjugated porous polymers has been demonstrated as an efficient strategy for improving their performance as heterogeneous photocatalysts. ${ }^{17,36}$ TTD-based polymers with weak electron donors may prevent the fast recombination of excitons favouring the formation of a triplet state. Studies to investigate the potential of these TTD-based CPPs as heterogeneous photocatalysts are underway and will be the subject of a future publication.

\section{Conclusions}

In summary, three narrow bandgap conjugated porous polymers (CPPs) have been synthesized by direct arylation polymerization of thiophene-flanked thienothiadiazole (TTD) - a low bandgap electron-accepting building block - with polytropic aryl bromides of various geometries. Direct arylation coupling between TTD and a series of mono-brominated aryls resulted in small molecular D-A-D triads in high yields, suggesting the relatively high reactivity and regioselectivity of TTD. Under the same polymerization conditions, the morphologies and porosities of the CPPs were determined by the structure of the aryl monomers. Specifically, DAP coupling between TTD and 1,3,5tribomobenzene resulted in fibrillar networks that showed significantly higher porosity than the other two CPPs with 2,2',7,7'-tetrabromo-9,9'-spirobifluorene and tris(4-bromophenyl)amine as comonomers, respectively. The bandgaps of the CPPs were determined by the $\mathrm{D}-\mathrm{A}$ intramolecular charge transfer. Among the three CPPs, CPP-2 composed of TTD and triphenylamine as repeating units showed the narrowest bandgap below $1.3 \mathrm{eV}$ due to the largest extent of $\mathrm{D}-\mathrm{A}$ charge transfer. These experimental results demonstrate that a rational selection of monomers enables the synthesis of a series of narrow bandgap conjugated porous polymers with tunable porosities, morphologies and optoelectronic properties.

\section{Acknowledgements}

M. W. is grateful for the funding support by a start-up grant (M4080992.120) of the Nanyang Assistant Professorship from the Nanyang Technological University, and AcRF Tier 1 (M4011061.120, RG49/12) from the Ministry of Education -

Table 2 Summary of electrochemical properties of TTD-(1-3) and CPP-(1-3)

\begin{tabular}{llllllrr}
\hline Species & $E_{\text {ox }}^{\text {onset }}(\mathrm{V})$ & $E_{\text {red }}^{\text {onset }}(\mathrm{V})$ & $E_{\text {HOMO }}{ }^{a}(\mathrm{eV})$ & $E_{\mathrm{LUMO}}{ }^{b}(\mathrm{eV})$ & $E_{\mathrm{g}}^{\text {Elec }}(\mathrm{eV})$ & $\lambda_{\text {edge }}(\mathrm{nm})$ & 838 \\
\hline TTD-1 & 0.739 & -0.694 & -5.54 & -4.11 & 1.43 & 1.30 & 898 \\
TTD-2 & 0.592 & -0.701 & -5.39 & -4.09 & -4.13 & 1.39 & 855 \\
TTD-3 & 0.724 & -0.671 & -5.52 & -4.07 & 1.41 & 1.38 \\
CPP-1 & 0.681 & -0.726 & -5.48 & -4.11 & 1.34 & 1.45 \\
CPP-2 & 0.652 & -0.695 & -5.45 & -4.03 & 1.51 & - \\
CPP-3 & 0.739 & -0.774 & -5.54 & $\mathrm{eV})$ & $943^{c}$
\end{tabular}

${ }^{a} E_{\mathrm{HOMO}}=-\left(E_{\mathrm{OX}}^{\text {onset }}+4.8\right) \cdot{ }^{b} E_{\mathrm{LUMO}}=-\left(E_{\mathrm{red}}^{\text {onset }}+4.8\right) \cdot{ }^{c}$ Optical bandgaps of CPP-1 and CPP-3 were determined by a rough estimation of the onset wavelengths. Onset of CPP-2 is too steep for determination of an onset wavelength. 
Singapore. Mass spectroscopy was carried out in the Proteomics and Mass Spectroscopy Services facility, School of Biological Sciences, Nanyang Technological University. H. B. gratefully acknowledges the Ph.D. research scholarships from Nanyang Technological University.

\section{Notes and references}

1 R. Dawson, A. Cooper and D. Adams, Prog. Polym. Sci., 2012, 37, 530-563.

2 Q. Liu, Z. Tang, M. Wu and Z. Zhou, Polym. Int., 2013, 63, 381-392.

3 N. McKeown and P. Budd, Macromolecules, 2010, 43, 51635176.

4 A. Slater and A. Cooper, Science, 2015, 348, 8075.

5 A. Thomas, Angew. Chem., Int. Ed., 2010, 49, 8328-8344.

6 Z. Xiang and D. Cao, J. Mater. Chem. A, 2013, 1, 26912718.

7 R. Dawson, A. Cooper and D. Adams, Polym. Int., 2013, 62, 345-352.

8 S. Hug, L. Stegbauer, H. Oh, M. Hirscher and B. Lotsch, Chem. Mater., 2015, 27, 8001-8010.

9 (a) H. Ma, F. Li, P. Li, H. Wang, M. Zhang, G. Zhang, M. Baumgarten and K. Müllen, Adv. Funct. Mater., 2016, 26, 2025-2031; (b) S. Bandyopadhyay, P. Pallavi, A. Anil and A. Patra, Polym. Chem., 2015, 6, 3775-3780.

10 (a) C. Gu, N. Huang, J. Gao, F. Xu, Y. Xu and D. Jiang, Angew. Chem., Int. Ed., 2014, 53, 4850-4855; (b) L. Xiang, Y. Zhu, S. Gu, D. Chen, X. Fu, Y. Zhang, G. Yu, C. Pan and Y. Hu, Macromol. Rapid Commun., 2015, 36, 1566-1571.

11 Z. Wang, S. Ghasimi, K. Landfester and K. Zhang, Adv. Mater., 2015, 27, 6265-6270.

12 C. Gu, N. Huang, Y. Chen, L. Qin, H. Xu, S. Zhang, F. Li, Y. Ma and D. Jiang, Angew. Chem., Int. Ed., 2015, 54, 1359413598.

13 J. Luo, X. Zhang and J. Zhang, ACS Catal., 2015, 5, 22502254.

14 Y. Xu, S. Jin, H. Xu, A. Nagai and D. Jiang, Chem. Soc. Rev., 2013, 42, 8012-8031.

15 (a) J. Jiang, F. Su, A. Trewin, C. Wood, H. Niu, J. Jones, Y. Khimyak and A. Cooper, J. Am. Chem. Soc., 2008, 130, 7710-7720; (b) R. Dawson, A. Laybourn, Y. Khimyak, D. Adams and A. Cooper, Macromolecules, 2010, 43, 85248530 .

16 (a) Y. Xu, A. Nagai and D. Jiang, Chem. Commun., 2013, 49, 1591-1593; (b) Z. Xie, S. Yoon and S. Park, Adv. Funct. Mater., 2010, 20, 1638-1644.

17 K. Zhang, Z. Vobecka, K. Tauer, M. Antonietti and F. Vilela, Chem. Commun., 2013, 49, 11158.

18 B. Bonillo, R. Sprick and A. Cooper, Chem. Mater., 2016, 28, 3469-3480.

19 (a) J. Jiang, F. Su, A. Trewin, C. Wood, N. Campbell, H. Niu, C. Dickinson, A. Ganin, M. Rosseinsky, Y. Khimyak and A. Cooper, Angew. Chem., Int. Ed., 2007, 46, 8574-8578; (b) E. Stöckel, X. Wu, A. Trewin, C. Wood, R. Clowes,
N. Campbell, J. Jones, Y. Khimyak, D. Adams and A. Cooper, Chem. Commun., 2009, 212-214.

20 (a) J. Schmidt, M. Werner and A. Thomas, Macromolecules, 2009, 42, 4426-4429; (b) X. Yang, S. Yao, M. Yu and J. Jiang, Macromol. Rapid Commun., 2014, 35, 834-839.

21 G. Cheng, T. Hasell, A. Trewin, D. Adams and A. Cooper, Angew. Chem., Int. Ed., 2012, 51, 12727-12731.

22 P. Li, T. Schon and D. Seferos, Angew. Chem., Int. Ed., 2015, 54, 9361-9366.

23 Y. Zhu, H. Long and W. Zhang, Chem. Mater., 2013, 25, 1630-1163.

24 (a) J. Weber, Q. Su, M. Antonietti and A. Thomas, Macromol. Rapid Commun., 2007, 28, 1871-1876; (b) M. Liebl and J. Senker, Chem. Mater., 2013, 25, 970-980. 25 (a) A. Facchetti, L. Vaccaro and A. Marrocchi, Angew. Chem., Int. Ed., 2012, 51, 3520-3523; (b) K. Wang and M. F. Wang, Curr. Org. Chem., 2013, 17, 999-1012; (c) L. Mercier and M. Leclerc, Acc. Chem. Res., 2013, 46, 1597-1605; (d) K. Okamoto, J. Zhang, J. Housekeeper, S. Marder and C. Luscombe, Macromolecules, 2013, 46, 8059-8078; (e) S. Kowalski, S. Allard, K. Zilberberg, T. Riedl and U. Scherf, Prog. Polym. Sci., 2013, 38, 1805-1814; (f) A. Rudenko and B. Thompson, J. Polym. Sci., Part A: Polym. Chem., 2014, 53, 135-147; (g) P. Morin, T. Bura and M. Leclerc, Mater. Horiz., 2016, 3, 11-20.

26 (a) M. Sévignon, J. Papillon, E. Schulz and M. Lemaire, Tetrahedron Lett., 1999, 40, 5873-5876; (b) J. Hassan, E. Schulz, C. Gozzi and M. Lemaire, J. Mol. Catal. A: Chem., 2003, 195, 125-131; (c) Q. Wang, R. Takita, Y. Kikuzaki and F. Ozawa, J. Am. Chem. Soc., 2010, 132, 11420-11421; (d) W. Lu, J. Kuwabara and T. Kanbara, Macromolecules, 2011, 44, 1252-1255; (e) P. Berrouard, A. Najari, A. Pron, D. Gendron, P. Morin, J. Pouliot, J. Veilleux and M. Leclerc, Angew. Chem., Int. Ed., 2011, 51, 2068-2071.

27 (a) R. Matsidik, H. Komber, A. Luzio, M. Caironi and M. Sommer, J. Am. Chem. Soc., 2015, 137, 6705-6711; (b) J. Pouliot, B. Sun, M. Leduc, A. Najari, Y. Li and M. Leclerc, Polym. Chem., 2015, 6, 278-282; (c) P. Morin, T. Bura, B. Sun, S. Gorelsky, Y. Li and M. Leclerc, ACS Macro Lett., 2015, 4, 21-24.

28 X. Wang and M. Wang, Polym. Chem., 2014, 5, 5784-5792.

29 (a) K. Wang, G. Wang and M. Wang, Macromol. Rapid Commun., 2015, 36, 2162-2170; (b) X. Wang, K. Wang and M. Wang, Polym. Chem., 2015, 6, 1846-1855; (c) H. Bohra, J. Shao, S. Huang and M. Wang, Tetrahedron Lett., 2016, 57, 1497-1501; (d) J. Huang, K. Wang, S. Gupta, G. Wang, C. Yang, S. Mushrif and M. Wang, J. Polym. Sci., Part A: Polym. Chem., 2016, 54, 2015-2031; (e) J. Shao, G. Wang, K. Wang, C. Yang and M. Wang, Polym. Chem., 2015, 6, 6836-6844.

30 (a) D. Liu, Q. Chen, Y. Zhao, L. Zhang, A. Qi and B. Han, ACS Macro Lett., 2013, 2, 522-526; (b) A. Efrem, K. Wang, P. Amaniampong, C. Yang, S. Gupta, H. Bohra, S. Mushrif and M. Wang, Polym. Chem., 2016, 7, 4862-4866.

31 Q. Chen, J. Wang, F. Yang, D. Zhou, N. Bian, X. Zhang, C. Yan and B. Han, J. Mater. Chem., 2011, 21, 13554-13560. 
32 (a) C. Hua, A. Rawal, T. Faust, P. Southon, R. Babarao, J. Hook and D. D'Alessandro, J. Mater. Chem. A, 2014, 2, 12466; (b) D. Wang, L. Li, W. Yang, Y. Zuo, S. Feng and H. Liu, RSC Adv., 2014, 4, 59877-59884.

33 J. Weber, J. Schmidt, A. Thomas and W. Böhlmann, Langmuir, 2010, 26, 15650-15656.

34 (a) S. Ren, R. Dawson, D. Adams and A. Cooper, Polym. Chem., 2013, 4, 5585-5590; (b) B. Kim, N. Park, S. Lee, H. Kim and S. Son, Polym. Chem., 2015, 6, 7363-7367.
35 (a) X. Gong, M. Tong, Y. Xia, W. Cai, J. Moon, Y. Cao, G. Yu, C. Shieh, B. Nilsson and A. Heeger, Science, 2009, 325, 1665-1667; (b) J. Yuen, R. Kumar, J. Seifter, S. Valouch, D. Zakhidov, D. Moses, U. Lemmer, A. Heeger and F. Wudl, J. Am. Chem. Soc., 2011, 133, 19602-19605.

36 (a) Z. Wang, S. Ghasimi, K. Landfester and K. Zhang, Chem. Commun., 2014, 50, 8177; (b) K. Zhang, D. Kopetzki, P. Seeberger, M. Antonietti and F. Vilela, Angew. Chem., Int. Ed., 2013, 52, 1432-1436. 